\title{
Mean Field Dynamo Saturation: Toward Understanding Conflicting Results
}

\author{
Eric G. Blackman \\ Dept. of Physics $\&$ Astronomy, University of Rochester, NY 91125
}

George B. Field

Harvard-Smithsonian Center for Astrophysics, Cambridge, MA 02138

\begin{abstract}
Mean field dynamos may explain the origin of large scale magnetic fields of galaxies, but controversy arises over the extent of dynamo quenching by the growing field. Here we explain how apparently conflicting results may be mutually consistent, by showing the role of magnetic helicity conservation and boundary terms usually neglected. We estimate the associated magnetic energy flowing out of the Galaxy but emphasize that the mechanism of field escape needs to be addressed.
\end{abstract}

\section{Field Growth and Constraining the Turbulent EMF}

Unlike the turbulent amplification of small scale magnetic energy to near equipartition with kinetic energy spectrum, mean field dynamo (MFD) action (Parker 1979, Kulsrud 1999) on scales larger than the turbulent input scale is controversial (c.f. Field et al. 1999). The MFD equation is $\partial_{t} \overline{\mathbf{B}}=\nabla \times\langle\mathbf{v} \times \mathbf{b}\rangle+\lambda \nabla^{\mathbf{2}} \overline{\mathbf{B}}+$ $\nabla \times(\overline{\mathbf{V}} \times \overline{\mathbf{B}})$, with the turbulent $\mathrm{EMF}\langle\mathbf{v} \times \mathbf{b}\rangle=\alpha \overline{\mathbf{B}}-\beta \nabla \times \overline{\mathbf{B}}$, and pseudoscalar $\alpha$ and scalar $\beta$. The survival of $\langle\mathbf{v} \times \mathbf{b}\rangle$ when the magnetic backreaction is included controls the efficiency of MFD. Blackman \& Field (2000a) used Ohm's law and mean field theory (e.g. $\mathbf{B}=\mathbf{b}+\overline{\mathbf{B}} ;\langle\mathbf{b}\rangle=0$ ) to constrain the dynamic value of $\langle\mathbf{v} \times \mathbf{b}\rangle$ analytically. Deriving $\langle\mathbf{v} \times \mathbf{b}\rangle \cdot \overline{\mathbf{B}} / \mathbf{c}=-\eta\langle\mathbf{j} \cdot \mathbf{b}\rangle+\langle\mathbf{e} \cdot \mathbf{b}\rangle$ and then expanding the fluctuating electric field $\mathbf{e}$ into its potentials, gives $\langle\mathbf{e} \cdot \mathbf{b}\rangle=-\partial_{t}\langle\mathbf{a} \cdot \mathbf{b}\rangle / 2 c+\nabla \cdot\langle\mathbf{a} \times \mathbf{e}-\phi \mathbf{b}\rangle$. Thus, for $\langle\mathbf{v} \times \mathbf{b}\rangle$ not to be resistively limited, there must be time variation of $\langle\mathbf{a} \cdot \mathbf{b}\rangle$, or non-vanishing boundary terms. When such terms vanish, helical turbulence without mean field gradients gives $\alpha \leq(b / \bar{B})^{2} \alpha_{0} / R_{m}$, where $\alpha_{0}$ is the kinematic value of $\alpha$, and $R_{m}$ is the magnetic Reynolds number. There is thus an ambiguity in interpreting all existing numerical experiments suggesting $\alpha$ quenching (e.g. Cattaneo \& Hughes 1996); the quenching might not be dynamical, but may be due to boundary conditions.

\section{Magnetic Helicity Escape, Dynamo Action, and Coronal Activity}

The above result highlights the role of total magnetic helicity $H^{M}=\int_{V} \mathbf{A} \cdot \mathbf{B} d^{3} x$ (Elsässer 1956), where $V$ is a volume of integration, and $\mathbf{A}$ is the vector potential. That MFD growth involves a magnetic helicity inverse cascade was demonstrated 
by Pouquet et al. (1976). The $\alpha$ effect conserves $H^{M}$ by pumping a positive (negative) amount to scales $>L$ (the outer turbulent scale) and a negative (positive) amount to scales $\ll L$. Brandenburg's (2000) simulations confirm this inverse cascade and the role of $H^{M}$ conservation.

A large-scale field can be generated only as fast as $H^{M}$ can be removed or dissipated given the upper limit on $H^{M}$ at small scales resulting from the realizability condition for the spectral density of $H^{M}$. Presently, simulations have invoked boundary conditions for which the growth of large scale field is resistively limited. Large $R_{m}$ systems must rely on open boundary conditions. To see this, note that $H^{M}$ satisfies $\partial_{t}(\mathbf{A} \cdot \mathbf{B})+c \nabla \cdot(\mathbf{E} \times \mathbf{A}+\phi \mathbf{B})=-2 c \mathbf{E} \cdot \mathbf{B}$ where $\mathbf{E}=-\mathbf{V} \times \mathbf{B} / c$. Consider two cases. (1): The mean scale $=$ universal scale, or the integration is over periodic boundaries. Then boundary terms vanish, so $\partial_{t}\langle\mathbf{A} \cdot \mathbf{B}\rangle=-2 c\langle\mathbf{E} \cdot \mathbf{B}\rangle=-2 c \overline{\mathbf{E}} \cdot \overline{\mathbf{B}}-2 c\langle\mathbf{e} \cdot \mathbf{b}\rangle=\eta\langle\mathbf{J} \cdot \mathbf{B}\rangle$ and $\partial_{t}(\overline{\mathbf{A}} \cdot \overline{\mathbf{B}})=-2 c \overline{\mathbf{E}} \cdot \overline{\mathbf{B}} ; \partial_{t}\langle\mathbf{a} \cdot \mathbf{b}\rangle=-2 c\langle\mathbf{e} \cdot \mathbf{b}\rangle$. Dynamo action is resistively limited. (2): The system's (e.g. Galaxy or Sun) mean volume $V \ll$ universal volume. Here we must use the gauge invariant relative helicity $H_{R}^{M}$ inside and outside of the spherical or disk rotator (Berger \& Field 1984). The integral over the the universal volume then satisfies $\partial_{t} \int_{U} \mathbf{A} \cdot \mathbf{B} d^{3} x=\partial_{t} H_{R, \text { in }}^{M}+\partial_{t} H_{R, \text { out }}^{M}=-2 c \int_{U} \mathbf{E}$. $\mathbf{B} d^{3} x \simeq 0$. The formulae for the $H_{R}^{M}$ of the mean and fluctuating quantities inside the rotator are $\partial_{t} H_{R, i n}(\overline{\mathbf{B}})=-2 c \int_{i n} \overline{\mathbf{E}} \cdot \overline{\mathbf{B}} d^{3} x+2 c \int_{S_{i n}}\left(\overline{\mathbf{A}}_{p} \times \overline{\mathbf{E}}\right) \cdot d \mathbf{S}$ and $\partial_{t} H_{R, i n}(\mathbf{b})=-2 c \int_{i n}\langle\mathbf{e} \cdot \mathbf{b}\rangle d^{3} x+2 c \int_{S_{i n}}\left\langle\mathbf{a}_{p} \times \mathbf{e}\right\rangle \cdot d \mathbf{S}$. In a steady state, $\partial_{t} H_{R, \text { in }}=0=\partial_{t} H_{R, \text { out }}$. This and $\mathbf{E} \cdot \mathbf{B} \cong 0$ imply that the above surface terms must be equal and opposite. Moreover, the surface term balances the $\overline{\mathbf{E}} \cdot \overline{\mathbf{B}}$ term in the $\partial_{t} H_{R, i n}^{M}$ equation. The boundary term can thus allow for a significant turbulent EMF because the latter is contained in $\overline{\mathbf{E}} \cdot \overline{\mathbf{B}}$. Dynamo action unrestricted by resisitivity is possible only in case (2). This is consistent with Pouquet et al. (1976) and Brandenburg (2000).

If $H^{M}$ flows through the boundary, then so does magnetic energy. Blackman \& Field (2000b) showed that a typical minimum power leaving a MFD system is given by $\dot{E}^{M} \geq k_{\min }\left|\dot{H}^{M}\right| / 8 \pi \simeq k_{\min }\left|\left\langle\alpha \bar{B}^{2}\right\rangle\right| V / 6$. Dynamos in the Sun, accretion disks, and the Galaxy would then lead to a net escape of magnetic energy and small and large scale magnetic helicity. Coronal activity from the emergence and dissipation of helical magnetic flux is thus a generic prediction of the MFD, and is observed directly in the Sun (cf. Pevtsov et al. 1999). For the Galaxy, $\dot{E}^{M} \gtrsim\left(\pi R^{2}\right) \alpha \bar{B}^{2} \sim 10^{40}(R / 12 \mathrm{kpc})^{2}\left(\alpha / 10^{5} \mathrm{~cm} / \mathrm{s}\right)(\bar{B} / 5 \mu \mathrm{G})^{2} \mathrm{erg} / \mathrm{s}$, in each hemisphere. Blackman \& Field (2000b) discuss how this may be consistent with energy input rates required by Savage (1995) and Reynolds et al. (1999).

\section{Open Questions}

A MFD unlimited by resistivity requires the helicity to flow through the boundary. However, even if the boundary conditions are open, it may be that in a real system one may have to include the dynamics of buoyancy or winds to demonstrate fully the non-resistive MFD (e.g. Moss et al. 1999). The physics of the effective turbulent diffusion at the boundary may not be the same as the turbulent diffusion inside the rotator. Note that turbulent diffusion of the mean magnetic field (not necessarily the actual field) across the boundary is also re- 
quired to maintain a quadrupole field in the Galaxy with a net flux inside the disk. Similarly, the solar cycle requires net diffusion through the boundary. The flow of helicity would appeal to the same dynamics needed by these constraints.

The analytic and numerical studies that we have seen which show catastrophic suppression of the dynamo coefficients, or resistively limited dynamo action, either (1) invoke periodic boundary conditions, and/or (2) are 2D, or (3) do not distinguish between zeroth order isotropic components of the turbulence and the higher order anisotropic perturbations for a weak mean field (Blackman \& Field 1999). Thus there always seems to be an alternative explanation, and the observed suppression is then ambiguous as we have described. This does not mean that some of the physical concepts found in the strong suppression results are invalid, but just that they may be valid only for the restricted cases considered. For example, might the observation that, in the presence of a weak mean field, the local Lagrangian chaos properties of the flow are changed for turbulence in a periodic box (e.g. Cattaneo et al. 1996) have something to do with the boundary conditions? While the answer is not yet clear, it is relevant that although the helicity constraint is global, it also becomes a local constraint for any sub-volume of a homogeneous periodic box once the system is fully mixed.

\section{References}

Blackman, E. G. \& Field, G. B. 1999, ApJ, 521, 597

Blackman, E. G. \& Field, G. B. 2000a, ApJ, 534, 984

Blackman, E. G. \& Field, G. B. 2000b, MNRAS, in press, astro-ph/9912459

Berger M. C. \& Field G. B. 1984, JFM, 147, 133

Brandenburg, A. 2000, submitted to ApJ, astro-ph/0006186

Cattaneo, F., \& Hughes, D. W. 1996, Phys. Rev. E. 54, 4532

Cattaneo, F., Hughes, D. W., \& Kim E.-J. 1996, Phys.Rev.Lett, 76, 2057

Elsässer, W. M. 1956, Rev. Mod. Phys. 23, 135

Field, G. B., Blackman, E. G., \& Chou, H. 1999, ApJ, 513, 638

Kulsrud, R. 1999, ARA\&A, 37, 37

Moss D., Shukurov A., \& Sokoloff D. 1999, A\&A, 340, 120

Parker, E. N. 1979, Cosmical Magnetic Fields (Oxford: Clarendon Press)

Pevtsov, A., Canfield, R. C., \& Brown, M. R., eds. 1999, Magnetic Helicity in Space and Laboratory Plasmas (American Geophysical Union)

Pouquet, A., Frisch, U., \& Léorat, J. 1976, JFM, 77, 321

Reynolds R. J., Haffner L. M., \& Tufte S. L. 1999, ApJ, 525, 21

Savage B. D., 1995, in The Physics of the Interstellar Medium and Intergalactic Medium, A. Ferrara, C. F. McKee, C. Heiles, \& P. R. Shapiro eds., ASP Conf. Ser. vol 80 (San Francisco: PASP), 233 